\title{
Holographic 3DTV Displays Using Spatial Light Modulators
}

\author{
Metodi Kovachev ${ }^{1}$, Rossitza Ilieva ${ }^{1}$, Philip Benzie ${ }^{2}$, G. Bora Esmer ${ }^{1}$, \\ Levent Onural $^{1}$, John Watson ${ }^{2}$, and Tarik Reyhan ${ }^{1}$ \\ 1 Dept. of Electrical and Electronics Eng., Bilkent University, TR-06800 Ankara, \\ Turkey \\ ${ }^{2}$ University of Aberdeen, King's College, AB24 3FX, Scotland, UK
}

\subsection{Introduction}

All functional blocks of a 3DTV system, such as its capture, compression, transmission and display units, are all important for a successful end-to-end operation. However, there is no doubt that the display unit has a special impact since the viewer interacts directly with it. Construction of a 3D display unit, which generates a replica of a 3D scene with an acceptable quality, has been a primary goal for researchers for a long time $[1,2]$. Current 3D display implementations are usually based on stereoscopic or autostereoscopic technologies. However, a true 3D display unit, such as a holographic 3DTV display device, is much more desirable due to superior $3 \mathrm{D}$ visual quality they promise. Naturally, a dynamic holographic device is needed for video operation. Spatial light modulator (SLM) technology is one convenient alternative for achieving the dynamic holographic display. An SLM is an array of pixels where each pixel modulates the phase and amplitude of light transmitted through or reflected from it [3]. Recently, multi-mega-pixel SLMs that can be electronically driven by a digital video interface (DVI) or video graphics adapter (VGA) are developed [4, 42]. Although the developments in SLM technologies during the last decade brought us new opportunities, currently achievable SLM parameters are still not sufficient for a satisfactory 3D display quality.

Holography is based on representing and storing the 3D scene information as an interference pattern. Therefore, holographic recordings require a high spatial resolution. Various methods, such as compression of fringe patterns, generation of horizontal-parallax-only (HPO) holograms and computation of binary holograms have been proposed to reduce the bandwidth requirements [5]. If an SLM is going to be used as a holographic display unit, a large array size with a small pixel pitch is essential. Holograms may be captured directly from charge-coupled devices (CCDs) with a high spatial resolution and dynamic range, or may be generated by computers or other means. A successful wavefront reconstruction can be achieved if the hologram features 
match the SLM parameters such as pixel pitch, array size, pixel geometry, dynamic range, etc.

A survey on liquid crystal (LC) SLMs is given in the next section. After a discussion of some different methods of hologram generation by computation in Sect. 15.3, both digital and optical reconstructions from such generated holograms are compared in Sect. 15.4. Conclusions are presented at the end.

\subsection{Survey on Electro-optical Properties of LC Spatial Light Modulators}

SLMs, as promising devices for the holographic displays, are the subject of discussion in this section. Liquid crystal SLMs are electro-optical devices that can modulate transmitted or reflected light; they contain a two-dimensional array of discrete cells or pixels $[3,6]$. Each pixel contains a liquid crystal layer sandwiched between two electrodes on glass substrates and has birefringence depending on the applied voltage. The applied voltage modulates the phase difference between the ordinary ray and the extraordinary ray in the pixel cell. This is equivalent to a rotation of light polarization angle when the incident light is polarized at a $45^{\circ}$ angle with respect to the fast polarizing axis orientation of the LC. A polarization analyzer converts phase difference modulation to gray scale levels. One of the electrodes is usually common for all pixels in the SLM. A potential difference $V(\zeta, \eta)$, with respect to the common electrode can be applied at each pixel. Both electrodes may be transparent; or one transparent and one reflective (mirror) electrodes may be used depending on the design. The first case corresponds to the transmission mode and the second case to the reflection mode. Nowadays SLMs consist of more than a million pixels and any pixel may be addressed or driven independently. Pixel sizes vary from $7 \mu \mathrm{m}$ up to $19 \mu \mathrm{m}$, and the number of pixels can go up to $3840 \times 2048$. Pixel size depends on photolithographic and microelectronic technologies.

These pixels are usually square and arranged as a matrix with an aspect ratio of 4:3 or 16:9 for the standard and panoramic displays, respectively.

It is not physically possible for the entire surface to be active. The SLM matrix structure is geometrically determined by four parameters: these are pixel pitch $(\Delta \zeta$ and $\Delta \eta)$, and gap $\left(g_{x}\right.$ and $\left.g_{y}\right)$ along the $X$ and $Y$ directions (Fig. 15.1). Pixel pitch is the distance between the centers of neighboring pixels and the gap is the non-active area between two neighboring pixels. The ratio of the gap area and the active zone determines the fill factor of the SLM where common commercial fill factors being $>90 \%$. The gap has usually low transparency or low reflectivity and introduces an attenuation of the light. The ratio of the input and output light intensities determines the efficiency and it is about $50 \%$ to $75 \%$ for modern SLMs.

Transmissive SLMs are manufactured by using etching technology on a transparent substrate. The relief created this way acts as a phase grating and 


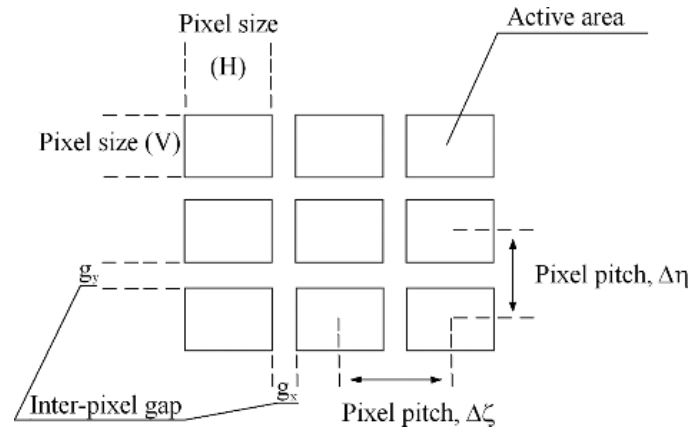

Fig. 15.1. Nomenclature for SLM pixel structure

creates multiple diffraction orders, as shown in Fig. 15.2, even when no voltage is applied. It is possible to use multiple diffraction orders as an advantage to enlarge the viewing zone or increase the effective SLM resolution [7, 8].

Reflective SLMs are manufactured by planar technology on a silicon substrate. Passive and active SLM elements are manufactured by diffusion and the relief on the silicon surface in this case has a depth less than the wavelength and introduces a small phase modulation in the reflected light. Because of this, the energy in high diffractive orders is much less than the energy in the zeroth or first orders of diffraction. LCoS (Liquid Crystal on Silicon) technology is usually a high resolution and a high fill factor process and pixel edges are

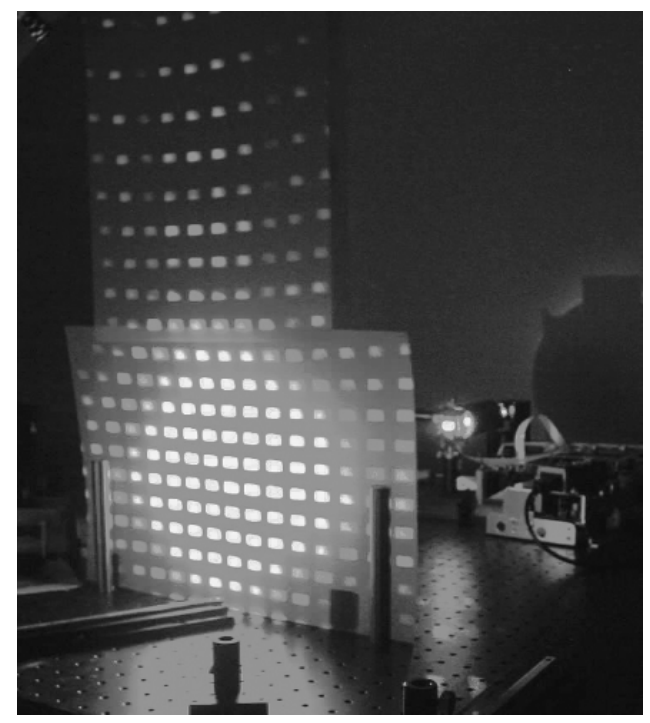

Fig. 15.2. Diffractive orders produced by an SLM illuminated by coherent laser source 
smooth. The electronic circuits that control the formation of the image are fabricated on the silicon chip which is coated with a highly reflective layer. The circuitry is behind the pixel, and therefore, does not create an obstruction along the light path.

The liquid crystal used in the cells of SLMs is a bi-refractive medium whose bi-refractive index depends on the applied voltage at the electrodes [9]. The voltage changes the refractive index of the liquid crystal in the extraordinary (fast) direction and the cell works as a phase-retarder under a suitably polarized light, obtained by a polarizer in front of the SLM. The polarizer is orientated $45^{\circ}$ toward the liquid crystal fast axis (Fig. 15.3). An analyzer after the SLM, orthogonally orientated with respect to the polarizer, transmits a portion of the light depending on the introduced retardation. In this case the SLM works in the amplitude-mostly mode. If the polarizer and analyzer are parallel to the fast axis of the liquid crystal then the SLM works in the phase-mostly mode.

Most SLMs can modulate the phase from zero to $2 \pi$ or $(-\pi$ to $+\pi)$ and some of them up to $3 \pi$ depending on the wavelength (for example: Holoeye Photonics: LC 2002, LC-R 2500, LC-R 768; HDTV Phase Only Panel HEO $1080 \mathrm{P})$. Characteristics of a Holoeye SLM are presented in Figs. 15.4.a 15.4.g. The phase modulation is measured relative to the linearly polarized reference beam. Intensity modulation is presented in relative units with respect to a chosen value from the corresponding gray level curve of $I(x, y)$.

Holoeye characteristics given in Figs. 15.4.a and 15.4.b show that the SLM behaviour depends strongly on the used polarizer. A polarizer with a better than 1:1100 attenuation ratio at $543 \mathrm{~nm}$ yields a high contrast ratio.

Input-output SLM characteristics are important for correct design and display of the computer generated holograms. The SLM characteristics for both modes depend on the polarizer and the analyzer orientations as shown in Figs. 15.4.c and 15.4.d. The phase and intensity modulation characteristics for an amplitude-mostly mode of transmissive SLM LC 2002 are shown in

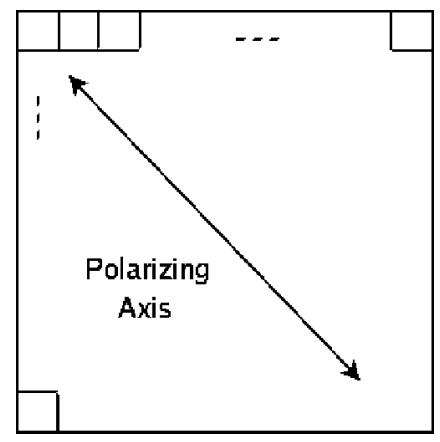

Fig. 15.3. Fast polarizing axis orientation of LC SLM 


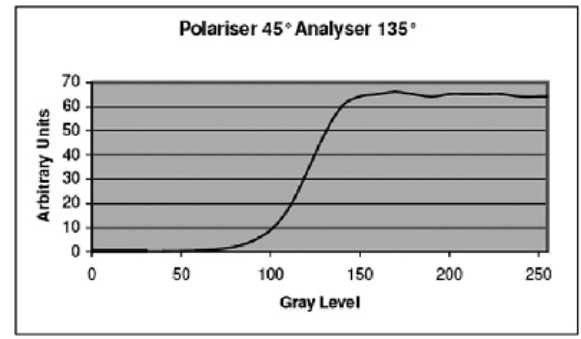

(a)

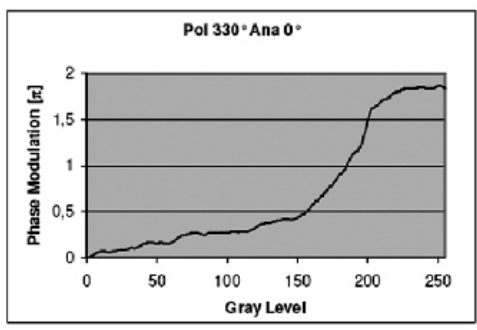

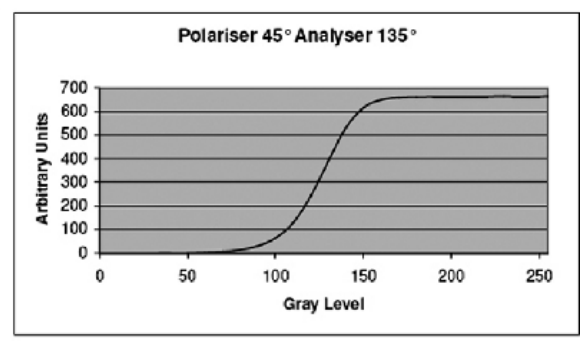

(b)

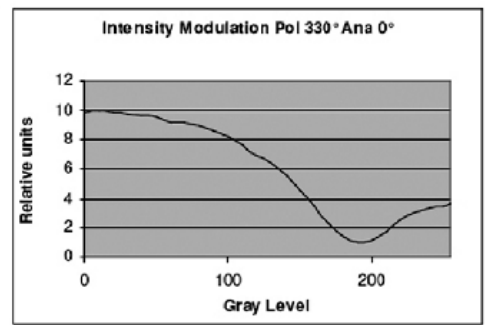

(c)
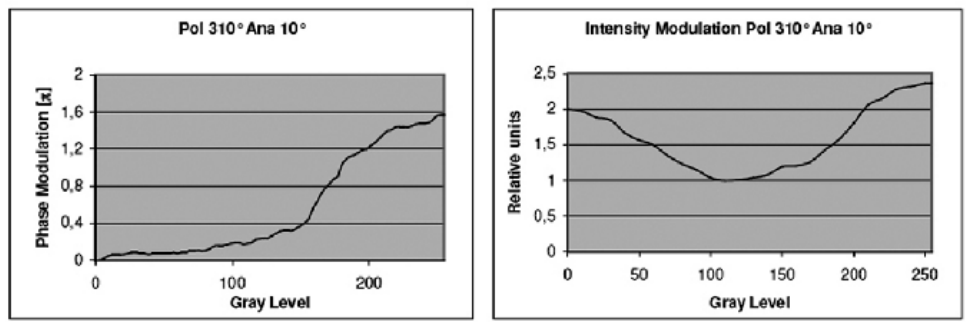

(d)

Fig. 15.4. Modulation characteristics of commercially available Holoeye SLMs. (a) Intensity modulation for LC 2002 at $543 \mathrm{~nm}$; (b) at $633 \mathrm{~nm}$; (c) phase and intensity modulation for different polarizer settings at $543 \mathrm{~nm}$; (d) at $633 \mathrm{~nm}$. (The phase modulation units are $\times \pi$ radians)

Fig. 15.4.c, for a polarizer orientation of $330^{\circ}$ and analyzer at $0^{\circ}$ with respect to the vertical axis of the SLM, at $543 \mathrm{~nm}$.

Phase and intensity modulation characteristics for Holoeye LC-R 768 reflective SLM for a polarizer orientation of $70^{\circ}$, and without an analyzer, at $543 \mathrm{~nm}$ and at $633 \mathrm{~nm}$ are shown in Figs. 15.4.e and 15.4.f, respectively. Therefore, the input polarization without an analyzer leads to an optimal light efficiency. Phase modulation for a polarizer orientation of $90^{\circ}$ and intensity modulation for polarizer and analyzer orientations of $90^{\circ}$ for phase-only SLM HEO $1080 \mathrm{P}(1920 \times 1080$ pixels $)$ at $633 \mathrm{~nm}$ are shown in Fig. $15.4 \mathrm{~g}$. It is 

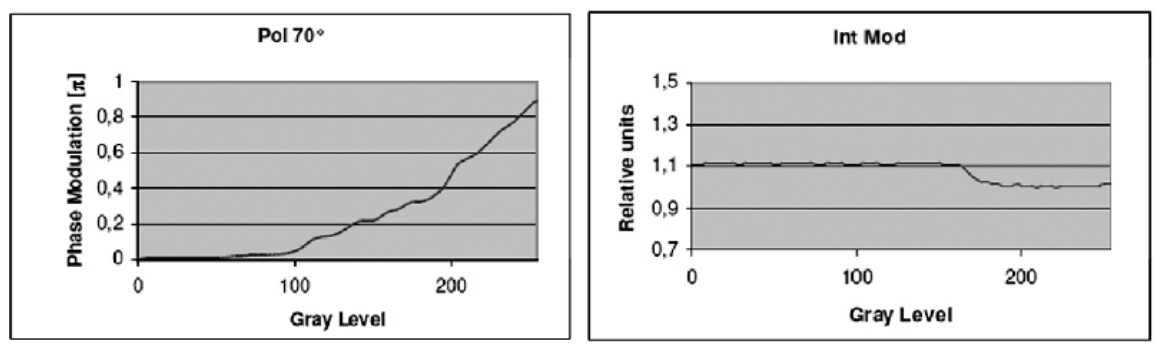

(e)
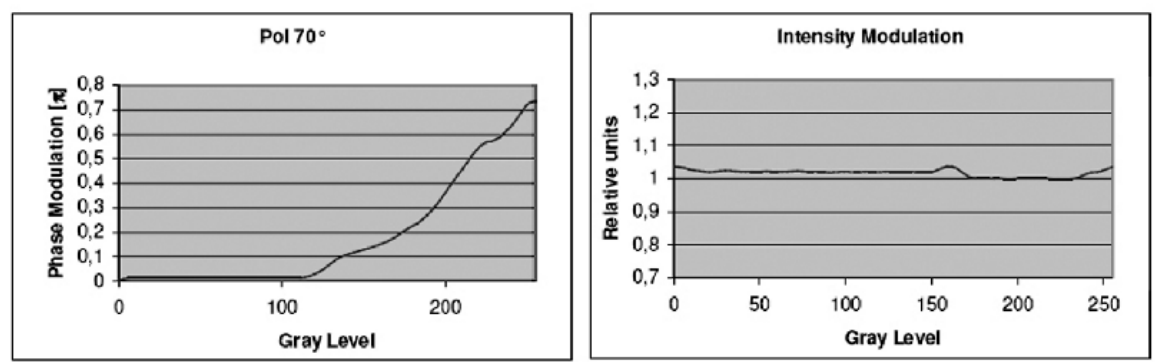

(f)
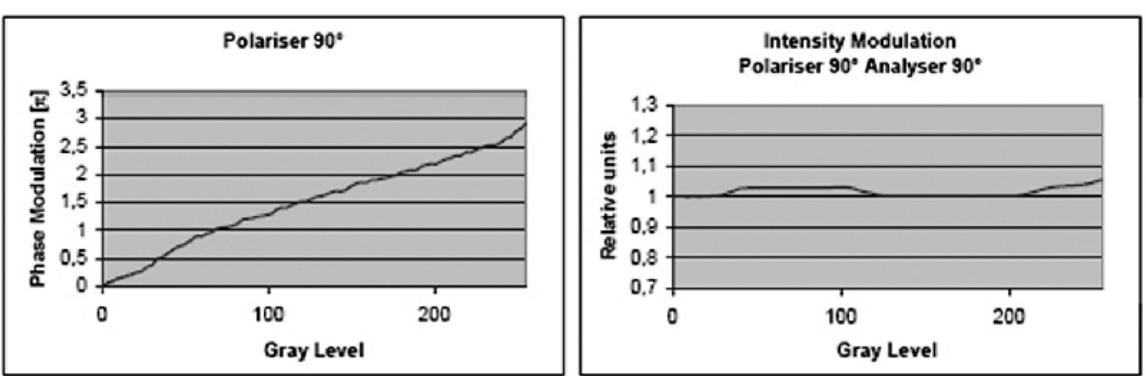

(g)

Fig. 15.4. (continued) Modulation characteristics of commercially available Holoeye SLMs. (e) phase and intensity modulation for LC-R 768 at $543 \mathrm{~nm}$; (f) at $633 \mathrm{~nm}$; (g) phase and intensity modulation for HEO $1080 \mathrm{P}$ at $633 \mathrm{~nm}$. (The phase modulation units are $\times \pi$ radians). (Courtesy of Stephan Osten from Holoeye Photonics $\mathrm{AG})$ 
seen that phase-mostly mode is always accompanied by a small amplitude modulation. Such characteristics are readily available on producers' (Holoeye Photonics, CRLO Displays Ltd., Displaytech Ltd.) documents.

Another important characteristic is the crosstalk between neighboring pixels of an SLM. Experiments with a Sony SLM LCX012BL, and a Holoeye LC 1004 [41] showed that the crosstalk is practically zero.

Most commercial SLMs suitable for computer generated holography (with a pixel count of greater than $1000 \times 1000$ ) have a minimum writing time which yields a frame rate of about $300 \mathrm{fps}$. Since the frame rate is rather high for video perception, single SLM color displays are possible by allocating the available frames to R, G, B color components in a time-multiplexed fashion. Alternatively, colour mixing can be achieved by using a simultaneous combination of transmissive and reflective SLMs.

\subsection{Generation of Holograms by Computation}

Methods and algorithms for computer generated holograms (CGHs) have been known for a long time [11, 28]. SLMs have been used as diffractive devices to reconstruct 3D images from CGHs $[4,11,12,13]$. Two common methods to compute the diffraction field due to a planar object in 3D space are the Rayleigh-Sommerfeld (R-S) diffraction integral and the Fresnel-Kirchoff diffraction formula. A comparison of R-S and Fresnel-Kirchhoff diffraction integrals is given by Lucke [14]. The Fraunhofer formula, which is also called the far field approximation, is valid for larger distances, $z>>\frac{k\left(x^{2}+y^{2}\right)_{\max }}{2}$, where $(x, y)$ represent the maximum extent of the object and $>>$ should be interpreted as "at least 15 times" [16].

The Fresnel diffraction formula should be used for smaller distances $[15,16]$. For a successful reconstruction, the size of the SLM, the reconstruction wavelength, the distance between the SLM and the image location, and many other parameters must be carefully considered. Three computational methods, which are based on R-S, Fresnel-Kirchhoff and the bipolar intensity formulas, are investigated in this Chapter.

The diffraction computation is usually a demanding process, therefore a number of algorithms have been employed to exploit redundancy and thus reduce the computation time $[5,17,18,21]$. For instance, Lucente et al. utilised a bipolar intensity method [5]. Ito et al. applied this method for reconstruction using LCoS SLMs [19, 20].

Classical methods for hologram computation use the wavefront propagation theory [22, 23, 24, 25]. R-S diffraction integral, Fresnel-Kirchhoff diffraction formula, and Fresnel approximation are some of well known scalar diffraction field calculation methods $[9,12,15,16,26,27]$. 


\subsubsection{Rayleigh-Sommerfeld Diffraction}

The scalar diffraction of monochromatic coherent light between two parallel planes in a homogeneous and linear medium can be expressed by the planewave decomposition (PWD). PWD and R-S diffraction integral are equivalent $[29,30]$. The difference between PWD and R-S diffraction integral is how they express the diffraction field relationship: former uses the frequency domain, whereas the latter defines the relationship in the spatial domain. PWD is used because of its simplicity in implementations.

The diffraction field relationship between the input and output fields by utilizing the plane wave decomposition is,

$$
U\left(x^{\prime}, y^{\prime}, z\right)=\int_{-2 \pi / \lambda}^{2 \pi / \lambda} \int_{-2 \pi / \lambda}^{2 \pi / \lambda} \Im[U(x, y, 0)] \exp \left[j\left(k_{x} \mathrm{x}+k_{y} \mathrm{y}\right)\right] \exp \left(k_{z} z\right) d k_{x} d k_{y}
$$

where $\Im$ is the 2D Fourier transform (FT) from $(x, y)$ domain to $\left(k_{x}, k_{y}\right)$ domain. The terms $k_{x}, k_{y}$ and $k_{z}$ are the spatial frequencies of the propagating monochromatic waves along the $x, y$ and $z$ axes, respectively. The spatial frequency $k_{z}$ can be expressed in terms of $k_{x}$ and $k_{y}$ as $k_{z}=\sqrt{k^{2}-k_{x}^{2}-k_{y}^{2}}$, where $k=2 \pi / \lambda$. The expression in (15.1) can be rewritten as:

$$
U\left(x^{\prime}, y^{\prime}, z\right)=4 \pi^{2} \Im^{-1}\left\{\Im[U(x, y, 0)] \exp \left(j \sqrt{k^{2}-k_{x}^{2}-k_{y}^{2}} z\right)\right\},
$$

where $\Im^{-1}$ is the inverse FT from $\left(k_{x}, k_{y}\right)$ domain to $(x, y)$ domain. Since we are dealing with propagating waves only, the diffraction field is band-limited. Moreover, to have a finite number of plane waves in the calculations, we work with periodic diffraction patterns. To obtain the discrete representation, (15.1) is sampled uniformly along the spatial axes with $x=n X_{s}, y=m X_{s}$ and $\mathrm{z}=p X_{s}$, where $X_{s}$ is the spatial sampling period, $n$ and $m$ are integers, and $p$ is a real variable. Uniform sampling is applied on the frequency domain with $k_{x}=2 \pi n^{\prime} / N X_{s}$ and $k_{y}=2 \pi m^{\prime} / N X_{s}$. The resultant discrete algorithm is

$$
U_{D}(n, m, p)=N D F T^{-1}\left\{D F T\left[U_{D}(n, m, 0)\right] H_{p}\left(n^{\prime}, m^{\prime}\right)\right\},
$$

where the term $H_{p}\left(n^{\prime}, m^{\prime}\right)=\exp \left(j 2 \pi \sqrt{\beta^{2}-n^{\prime 2}-m^{\prime 2}} p / N\right)$ and $\beta=N X_{s} / \lambda$. The discrete diffraction field $U_{D}(n, m, p)$ is:

$$
U_{D}(n, m, p)=U\left(n X_{s}, m X_{s}, p X_{s}\right) .
$$

Effects of sampling of the R-S diffraction field on the reconstructed image are discussed in [43].

\subsubsection{Fresnel-Kirchhoff Diffraction}

When the distance $z$ is sufficiently large, then the observer is said to be in the Fresnel diffraction region. The condition for distance $z$ is $[15,16]$ : 


$$
z>>\sqrt[3]{\frac{\pi}{4 \lambda}\left[\left(x^{\prime}-x\right)^{2}+\left(y^{\prime}-y\right)^{2}\right] \max }
$$

where $>>$ should be again interpreted as "at least 15 times". For instance, for an SLM with a $17.8 \mathrm{~mm}$ diagonal size, a viewing zone with the same size, and a pixel size of $12.1 \mu \mathrm{m}$, and for an illumination with a wavelength $\lambda=0.543 \mu \mathrm{m}$, the above condition becomes $z>0.530 \mathrm{~m}$.

The field over the hologram plane $U\left(x^{\prime}, y^{\prime}, z\right)$ according to Fresnel diffraction is $[16]$ :

$$
U\left(x^{\prime}, y^{\prime}, z\right)=\frac{\exp (j k z)}{j \lambda z} \iint U(x, y, 0) \exp \left\{j \frac{k}{2 z}\left[\left(x^{\prime}-x\right)^{2}+\left(y^{\prime}-y\right)^{2}\right]\right\} d x d y
$$

Equation 15.3 above is the convolution of the object function $U(x, y, 0)$ with the kernel $K(x, y, z)$ which is given by:

$$
K(x, y, z)=-\frac{j}{\lambda z} \exp (j k z) \exp \left(j k \frac{x^{2}+y^{2}}{2 z}\right) .
$$

For the moment, we will drop the constant terms in $(15.4)\left(-j / \lambda_{z} ; \exp (j k z)\right)$ for simplicity, and denote $U(x, y, 0)$ with $U(x, y)$. Now if we have a $2 \mathrm{D}$ discrete array with an overall dimensions $X$ and $Y$, (e.g. an SLM), then for the inner integral, with respect to $x$, we can write [12]

$$
\int_{-X / 2}^{+X / 2} U(x, y) \exp \left[j k \frac{\left(x^{\prime}-x\right)^{2}}{2 z}\right] d x=\int_{x_{1}}^{x_{2}} B d x+\int_{x_{2}}^{x_{3}} B d x+\int_{x_{3}}^{x_{4}} B d x+\ldots
$$

where,

$$
\begin{gathered}
B=U(x, y) \exp \left[j k \frac{\left(x^{\prime}-x\right)^{2}}{2 z}\right] \\
U(x, y)=U\left(x_{i}, y\right)=c_{i} ; \text { for } x_{i} \leq x \leq x_{i+1} \text { and } y=\sigma
\end{gathered}
$$

Here $c_{i}$ 's and $\sigma$ are constants. Thus we split the original integral into many integrals each defined over a single pixel and set the integral boundaries to coincide with the pixel boundaries. Therefore, over the area of a single pixel, the input field is constant; so it can be moved out of the integral:

$$
\int_{x_{i}}^{x_{i+1}} B d x=U\left(x_{i}, y\right) \int_{x_{i}}^{x_{i+1}} \exp \left[j k \frac{\left(x^{\prime}-x\right)^{2}}{2 z}\right] d x
$$

For the exponent argument and integral boundaries the following substitutions can be made: $\left(x-x^{\prime}\right) \sqrt{2} / \sqrt{\lambda z}=\tau ; d x=\sqrt{\lambda z} / \sqrt{2} d \tau ;\left.\tau\right|_{x=x_{i}}=\tau_{i}$. Each of the integrals in (15.5) can be written in terms of the Fresnel integral as, 


$$
\begin{aligned}
& \int_{\tau_{i}}^{\tau_{i+1}} \exp \left(\frac{j \pi}{2} \tau^{2}\right) d \tau=\int_{0}^{\tau_{i+1}} \exp \left(\frac{j \pi}{2} \tau^{2}\right) d \tau-\int_{0}^{\tau_{i}}\left(\frac{j \pi}{2} \tau^{2}\right) d \tau \\
& =C\left(\tau_{i+1}\right)+j S\left(\tau_{i+1}\right)-C\left(\tau_{i}\right)-j S\left(\tau_{i}\right),(1 \leq i \leq \mathrm{N}),
\end{aligned}
$$

where $C\left(\tau_{i}\right)$ and $S\left(\tau_{i}\right)$ are cosine and sine Fresnel integrals [9, 16]. Integrals along the $y$ direction can be calculated in the same way. If we take into account the relation between $\tau$ and $x$,

$$
\tau_{i-j}=\left(x_{i}-x_{j}\right) \frac{\sqrt{2}}{\sqrt{\lambda z^{\prime}}}
$$

and that assume that each integral above is multiplied by a piece of the input field corresponding to the interval $\left[x_{i}, x_{i+1}\right]$, we can write:

$$
\begin{gathered}
\frac{\sqrt{\lambda z}}{\sqrt{2}} U\left(x_{i}, y\right)\left[C\left(\tau_{(i+1)-j}\right)+j S\left(\tau_{(i+1)-j}\right)-C\left(\tau_{i-j}\right)-j S\left(\tau_{i-j}\right)\right], \\
(1 \leq i, j \leq N) .
\end{gathered}
$$

Therefore for the sum of integrals in (5) along the $x$-direction we obtain:

$$
\frac{\sqrt{\lambda z}}{\sqrt{2}} \sum_{i=1}^{N} U\left(x_{i}, y\right)\left[C\left(\tau_{(i+1)-j}\right)+j S\left(\tau_{(i+1)-j}\right)-C\left(\tau_{i-j}\right)-j S\left(\tau_{i-j}\right)\right] .
$$

This is the convolution in discrete form along the $x$-direction. In a similar way, for the $y$-direction we can write:

$$
\frac{\sqrt{\lambda z}}{\sqrt{2}} \sum_{k=1}^{M} U\left(x, y_{k}\right)\left[C\left(\sigma_{(k+1)-l}\right)+j S\left(\sigma_{(i+1)-l}\right)-C\left(\sigma_{k-l}\right)-j S\left(\sigma_{k-l}\right)\right],
$$

where $\left(y-y^{\prime}\right) \sqrt{2} / \sqrt{\lambda z}=\sigma ; d y=\sqrt{\lambda z} / \sqrt{2} d \sigma ;\left.\sigma\right|_{y=y_{i}}=\sigma_{i}$.

Combining the expressions for both $x$ and $y$ directions one can derive the $2 \mathrm{D}$ convolution in its discrete form as,

$$
\begin{aligned}
U\left(x_{j}^{\prime}, y_{l}^{\prime} ; z\right)= & -\frac{1}{2} \exp (j k z) \sum_{k=1}^{M} \sum_{i=1}^{N} U\left(x_{i}, y_{k} ; 0\right)\left\{\left[j C\left(\tau_{(i+1)-j}\right)+S\left(\tau_{(i+1)-j}\right)\right.\right. \\
& \left.-j C\left(\tau_{i-j}\right)-S\left(\tau_{i-j}\right)\right]\left[j C\left(\sigma_{(k+1)-l}\right)+S\left(\sigma_{(k+1)-l}\right)-j C\left(\sigma_{k-l}\right)\right. \\
& \left.\left.-S\left(\sigma_{k-l}\right)\right]\right\} .
\end{aligned}
$$

The kernel, expressed by the terms in curly brackets in (15.6), can be easily calculated by using standard algorithms [9]. This kernel takes into account the wavefront contribution of each pixel area in the diffraction pattern. The convolution can be calculated directly or by discrete Fourier transform.

The second step in calculation of a hologram is to add a reference beam co-linear to the propagation direction for an in-line (on-axis) hologram, or angular for an off-axis hologram. 


\subsubsection{Bipolar Intensity Method}

Now we will consider the bipolar intensity method, which derives its name from producing an interference pattern that has both positive and negative values.

In classical optical holography, we may consider that a hologram, $H$, consists of the combination of the complex valued wavefront of an object beam, $U$, and reference beam, $R$, onto the recording medium, as,

$$
H=|R+U|^{2}=|R|^{2}+|U|^{2}+U R^{*}+R U^{*}
$$

where $R=\left|a_{R}(x, y)\right| \exp \left\{j \varphi_{R}(x, y)\right\}$ and $U=\left|a_{U}(x, y)\right| \exp \left\{j \varphi_{U}(x, y)\right\}$ are the reference and object beams, respectively. The first term in the above expression is called the reference bias and it is a spatially invariant (DC) term. Second term denotes the object self-interference which is a spatially varying pattern. This term can cause distortion in the reconstruction process; fortunately this distortion is small for small objects and can be suppressed by having a tilted reference beam. Removal of these undesired terms helps in improving the computational efficiency and reduces the noise generated by the object beam. The final sinusoidal term describes the modulation of the object and reference beams and actually these are the terms which are computed as the hologram. Elimination of the first two terms results in an interference pattern with positive and negative intensities and hence the method is called the bipolar intensity method. Therefore, the intensity can be represented as:

$$
I_{\text {bipolar }}(x, y)=2\left|a_{U}(x, y)\right|\left|a_{R}(x, y)\right| \cos \left[\varphi_{R}(x, y)-\varphi_{U}(x, y)\right],
$$

where, $\varphi_{U}(x, y)$, represents the phase of the object beam. Here, we assume that the wavefront can be represented by a spherical wavefront and setting the scaling factor $2|U||R|$ to unity, and omitting $\varphi_{R}(x, y)$ term, which is taken as zero at the recording plane, we arrive at (15.7). This is the bipolar intensity method used for calculating holograms.

Intensity at each pixel location $\left(x_{\alpha}, y_{\alpha}\right)$ on the SLM can be defined as,

$$
I_{\text {bipolar }}\left(x_{\alpha}, y_{\alpha}\right)=\sum_{j-1}^{N o . p t s .} A_{j} \cos \left(\frac{2 \pi}{\lambda} \sqrt{\left(x_{\alpha}-x_{j}\right)^{2}+\left(y_{\alpha}-y_{j}\right)^{2}+z_{j}^{2}}\right) .
$$

where, $x_{j}, y_{j}, z_{j}$, are the real co-ordinate locations of the points on an object and $A_{j}$ is the amplitude of the corresponding point on the object.

During the calculation of (15.7) it is necessary to normalise the bipolar intensity so that all values are positive and can be written onto the SLM. This is easily done by adding a proper DC offset to all pixels. The dynamic range of the intensity levels are then quantized according to the dynamic range of the SLM. The resultant pixel values can be directly written onto the SLM. Equation (15.7) enables the reconstruction of 3D scenes described as object 
points and can be easily implemented into graphics commodity hardware for fast real-time computation of holograms [31].

LC or LCoS SLM can be used to display holograms $[4,11,12,13]$. Usually in-line holograms are reconstructed with LCoS SLMs. The advantage of the in-line reconstruction geometry is that the reference and object beams are collinear, thus the resolution requirements of the SLM are less demanding [32]. Due to the limited spatial bandwidth product offered by the SLM, it is only possible to reconstruct holograms with a limited viewing angle and spatial resolution.

A hologram can be also recorded directly by a CCD camera and then reconstructed numerically [33].

\subsection{Comparison of Numerical and Optical Reconstructions}

A program for computation of forward or backward propagated wavefront using Fresnel diffraction integral (15.6) is written [12]. A hologram is obtained by adding a reference beam to the forward propagated wavefront. In our case numerical or optical reconstructions from the generated holograms are obtained by using the complex conjugate of the reference beam and by backward propagating the field. If the reference beam itself was used, instead, then the virtual image would have been reconstructed. Holograms computed by the RayleighSommerfeld diffraction formula (15.2), and by bipolar intensity method (15.7), are also calculated, and reconstructions from these holograms are obtained. SLMs used during the optical reconstructions were LC for the transmission mode and LCoS for the reflection mode. The LC SLM, which is also commonly used in multimedia projectors, has a resolution of $1280 \times 720$ square pixels with a pitch of $12.1 \mu \mathrm{m}$ and a gap of about $1 \mu \mathrm{m}$. A $635 \mathrm{~nm}$ diode laser is used during the reconstruction.

For the LCoS SLM the resolution is $1900 \times 1200$ square pixels and the pitch is $8.1 \mu \mathrm{m}$; a He-Ne laser $(632.8 \mathrm{~nm})$ is used for illumination. The maximum diffraction angle for the LC SLM, determined by its pixel size, is $1.5^{\circ}$ and the minimum distance for a Gabor (in-line) hologram, to avoid overlapping of diffractive orders in the reconstructed image, is $350 \mathrm{~mm}$. The maximum diffraction angle for LCoS SLM is $2.2^{\circ}$.

Experimental setups for optical reconstructions, for the reflection and transmission modes, are shown in Fig. 15.5.

A Star Target is used as the object for computer simulations. This particular pattern is chosen to test the resolution of the overall process. Holograms of this object are calculated for a reconstruction distance of $800 \mathrm{~mm}$.

R-S in-line hologram, of the Star Target object given in Fig. 15.6, is shown in Fig. 15.7. In-line and off-axis Fresnel holograms of the same object are calculated using (15.6) and are shown in Figs. 15.8 and 15.9, respectively. 

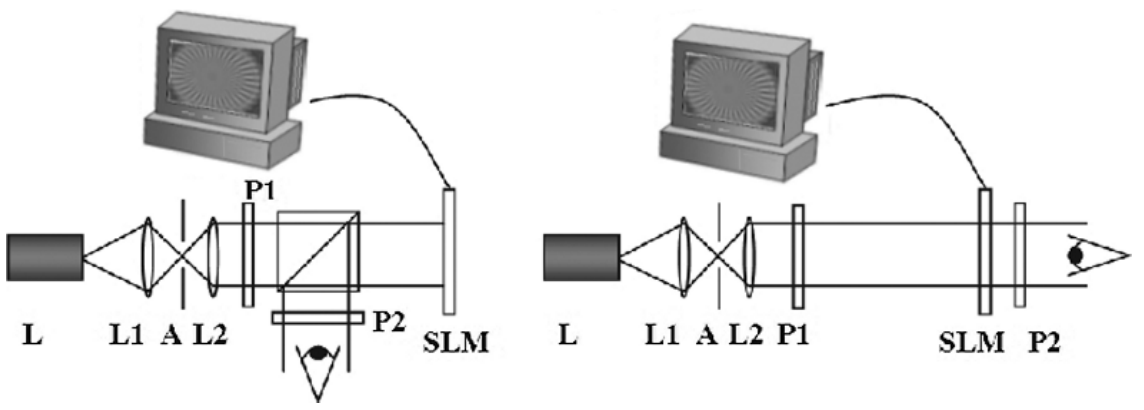

Fig. 15.5. Assembled experimental setups. L denotes the laser; L1 and L2 are the collimating lenses; A shows the pinhole; P1 is the polarizer, and P2 is the analyzer

Reconstructed images from Fresnel holograms (Figs. 15.8 and 15.9) by computer simulation are shown in Figs. 15.10 and 15.11. Diffracted field of the object at $800 \mathrm{~mm}$ distance is about two times the SLM size in both $x$ and $y$ directions. Reconstructed image, (Fig. 15.10), from Fresnel in-line hologram is corrupted because of the twin image, edge effects, and the periodicity. The implied periodicity of the original pattern is a result of using DFT algorithm in computation of convolution between the field $U(x, y)$ and the kernel $K(x, y, z)$. As seen from Fig. 15.11, the off-axis case overcomes the twin-image corruption, as expected.

Optically reconstructed images by a red $(635 \mathrm{~nm})$ laser diode are captured by a CCD camera (JenOptik AG type $11 \mathrm{MP}$ CCD); the results are shown in Figs. 15.12-15.15.

It is observed that the simulated (Figs. 15.10 and 15.11) and optically reconstructed (Figs. 15.12-15.15) Star Target images are well matched. A magnified portion of the reconstructed Star Target image from the off-axis hologram is shown in Fig. 15.12. The rays of the star are well reconstructed. The zeroth-order (directly transmitted) beam and reconstructed image in plus first order from the off-axis hologram are shown in Fig. 15.14. The image is

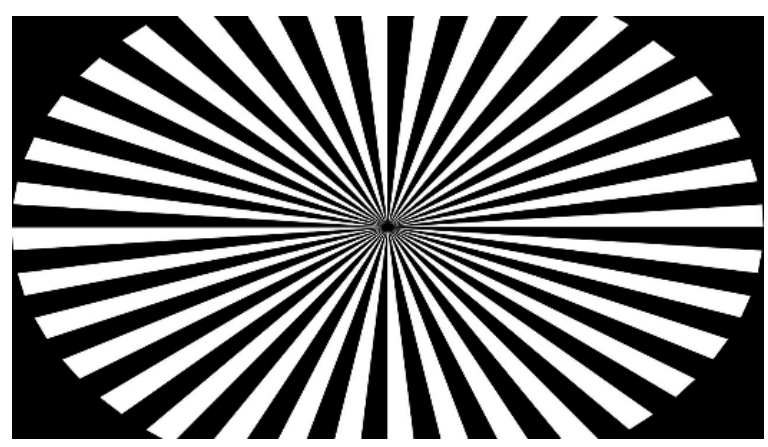

Fig. 15.6. Star Target object (First published by Springer[12]) 


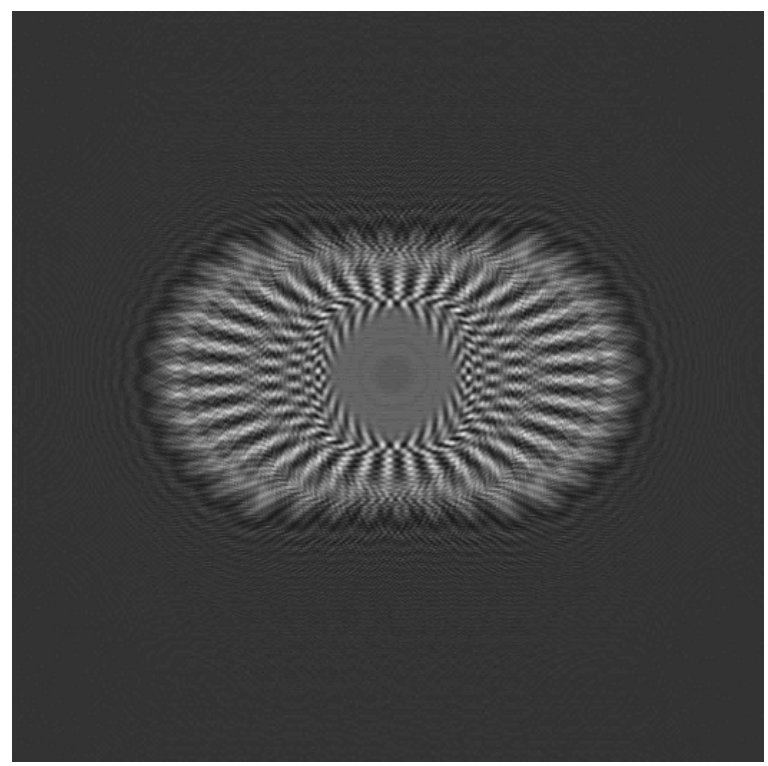

Fig. 15.7. R-S in-line hologram of the Star Target object shown in Fig. 15.6

situated diagonally to the zeroth order. The minus first order is the virtual image on the other side of the diagonal. The qualities of reconstructed images by the LC SLM from the in-line R-S (Fig. 15.15) and Fresnel (Fig. 15.13) holograms are visually similar.

In the series of reconstructed images there is a clearly perceivable difference between the in-line and off-axis image qualities, the off-axis being superior. However the angle between the object and reference beams has to be carefully chosen, so that the reconstructed object pattern does not overlap with the other diffracted orders of the SLM. It must be taken into account that this

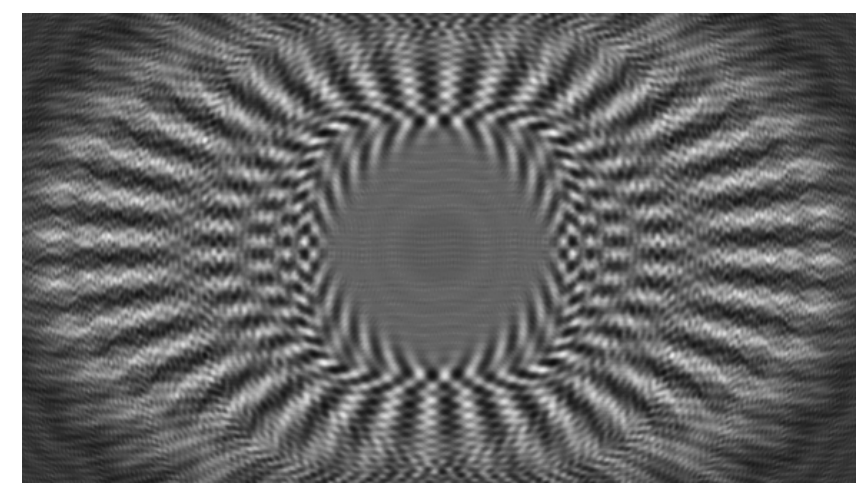

Fig. 15.8. Fresnel in-line hologram of the Star Target object shown in Fig. 15.6 


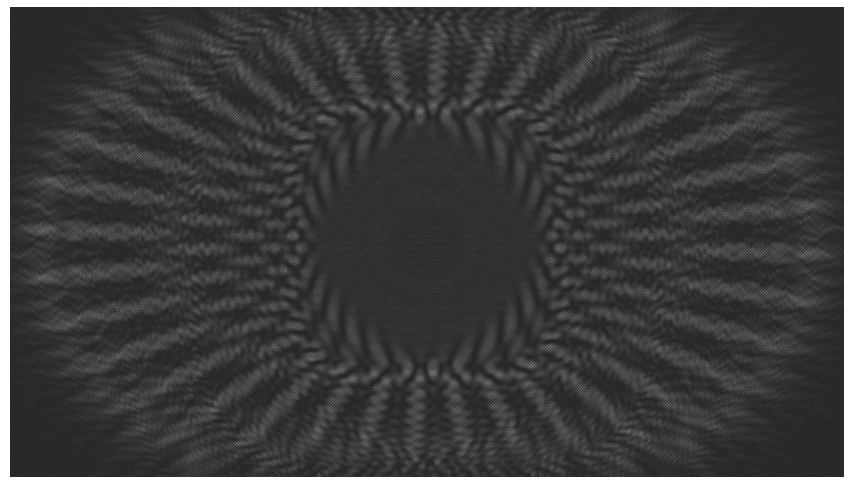

Fig. 15.9. Fresnel off-axis hologram of the Star Target object shown in Fig. 15.6 (First published by Springer [12])

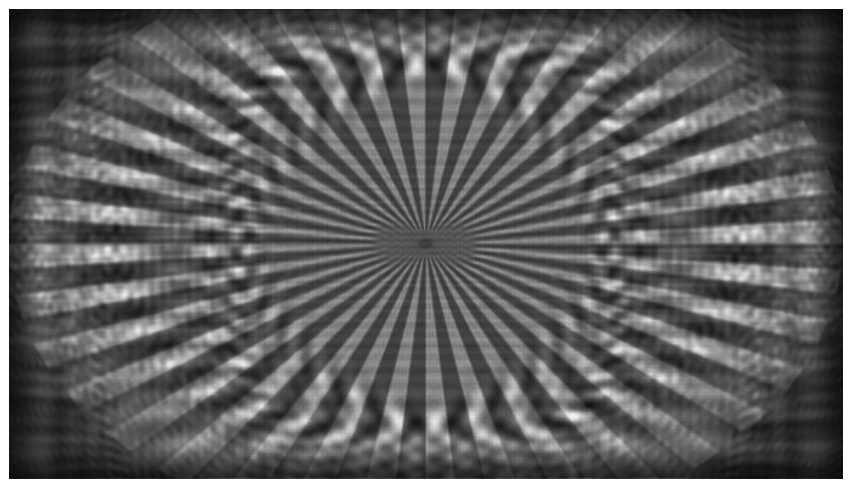

Fig. 15.10. Computer reconstructed image from the Fresnel in-line hologram shown in Fig. 15.8

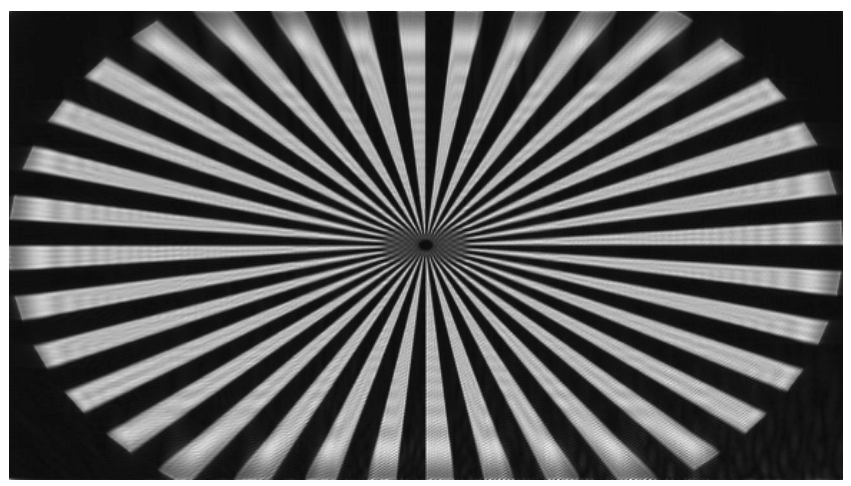

Fig. 15.11. Computer reconstructed image from the off-axis hologram shown in Fig. 15.9 (First published by Springer [12]) 


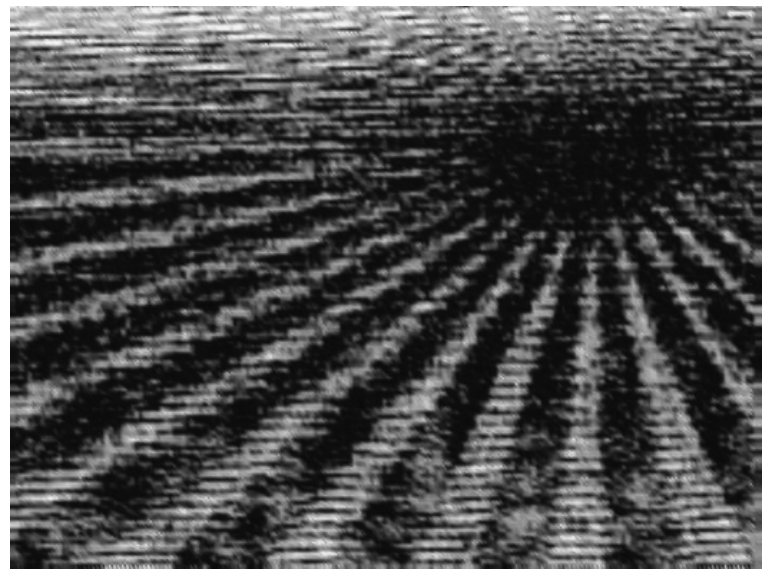

Fig. 15.12. Optically reconstructed and magnified image from the off-axis hologram shown in Fig. 15.9 by using a red laser (First published by Springer [12])

angle is restricted due to the pixel size. The optimal angle for the used LC SLM is $0.76^{\circ}$ when the reconstruction distance from the SLM is $800 \mathrm{~mm}$. Figure 15.16 illustrates an example when the chosen angle of the reference beam is $0.55^{\circ}$ which is less than the optimal, and thus the reconstructed object overlaps with the zeroth order. It is also possible that real and virtual images of the neighboring SLM orders may also overlap. This occurs when the angle is larger than the optimal.

It is worthwhile mentioning that good results are obtained by in-line holograms in case when the object is much smaller than the SLM size and has white letters over a black background. Such an example is shown in Figs. 15.17-15.19, where the object is in Fig. 15.17, the computed hologram

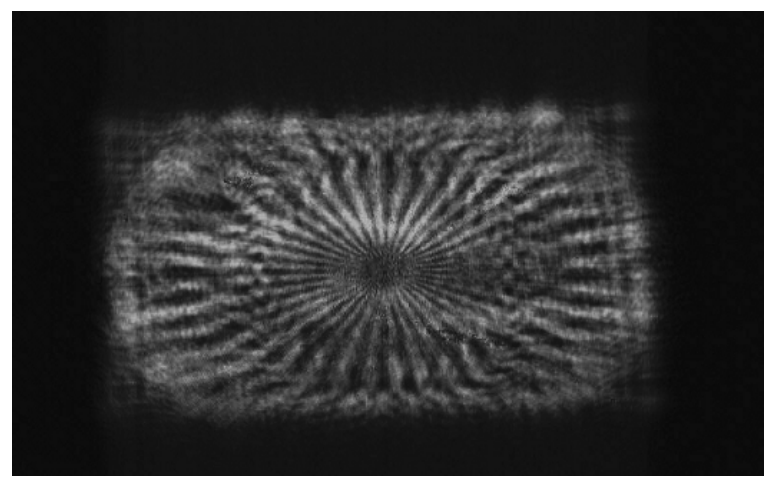

Fig. 15.13. Optically reconstructed image from the Fresnel in-line hologram shown in Fig. 15.8 


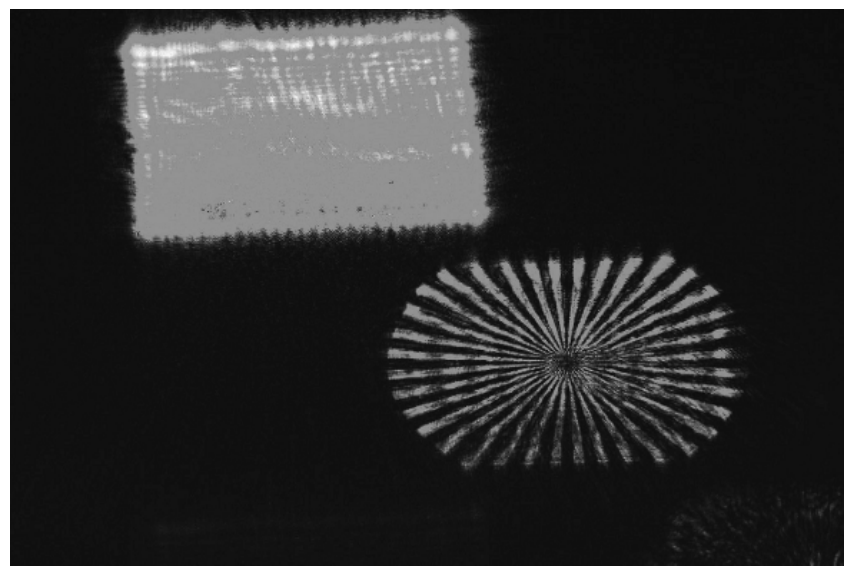

Fig. 15.14. Optically reconstructed image from the Fresnel off-axis hologram is shown in Fig. 15.9

is in Fig. 15.18 and the optically reconstructed image is in Fig. 15.19. To reduce the distortions caused by the first diffraction order and the DC term, the reconstructed image at the second diffraction order is taken.

As already mentioned, a good and correct reconstruction can be achieved if the parameters of the SLM and the computed hologram match. However, it is highly desirable to compute the holograms in a generic fashion without considering the eventual physical SLM parameters; reconstructions using different SLMs from the same hologram will provide flexibility. In an attempt to experimentally check the possible degradation in quality when different SLMs are used during the reconstruction, we computed a hologram for a

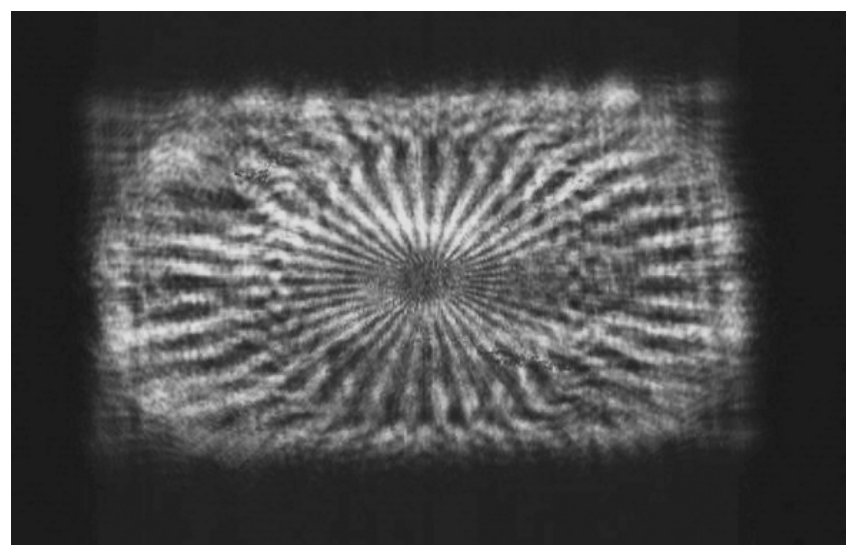

Fig. 15.15. Optically reconstructed image from R-S in-line hologram shown in Fig. 15.7 


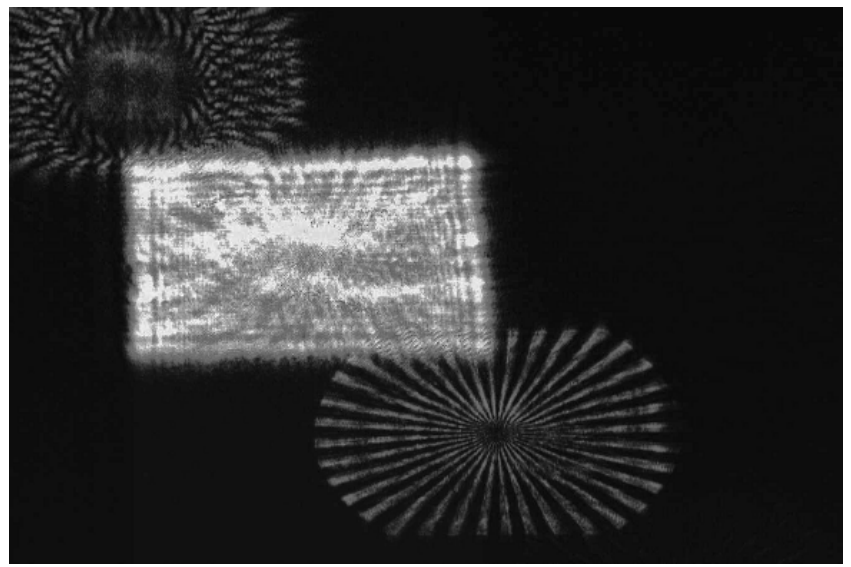

Fig. 15.16. Optically reconstructed image when the reference beam angle is less than optimal

\section{DTV}

Fig. 15.17. "3DTV" Object

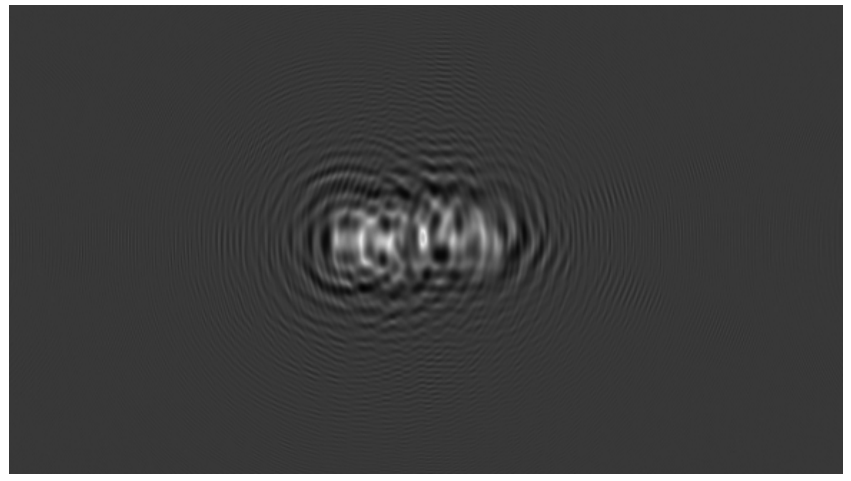

Fig. 15.18. In-line R-S hologram of Fig. 15.17 


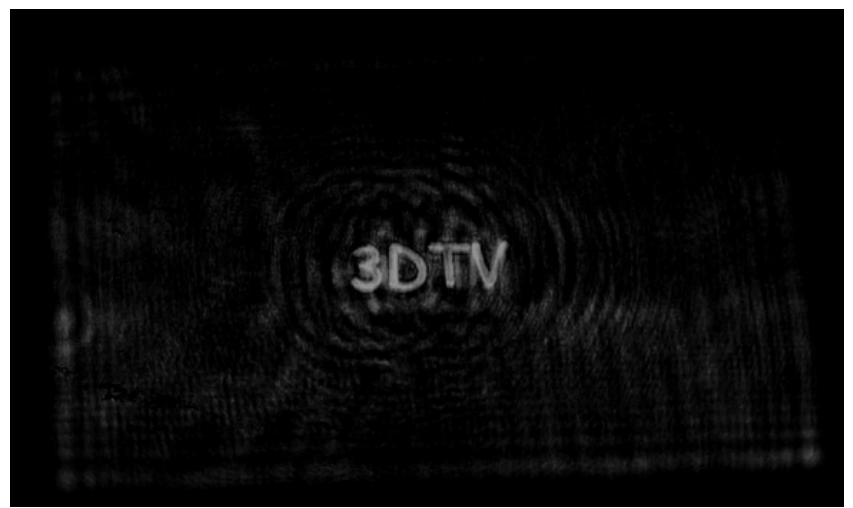

Fig. 15.19. Reconstructed image from Fig. 15.18

given SLM, but used a different SLM in addition to the original one, for the reconstruction. An in-line hologram (Fig. 15.21) of the artificially created "sine-wave" object, computed using the bipolar method (15.7) for the LCoS SLM, is shown in Fig. 15.20. An off-axis hologram of the Star Target object, which is calculated by the Fresnel off-axis method (15.6) for the LC SLM is shown in Fig. 15.9. Then, optical reconstructions using both LCoS and LC SLMs are conducted. Obtained results are shown in Figs. 15.22-15.24.

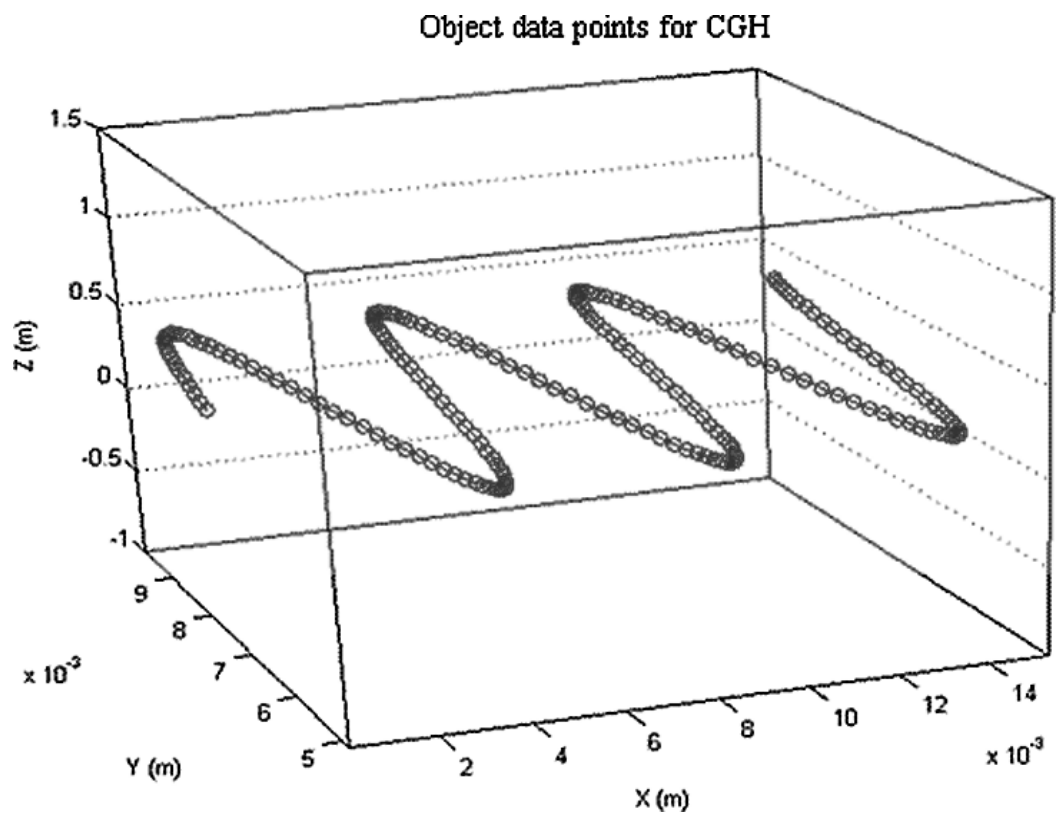

Fig. 15.20. The artificially generated "sine-wave" object 


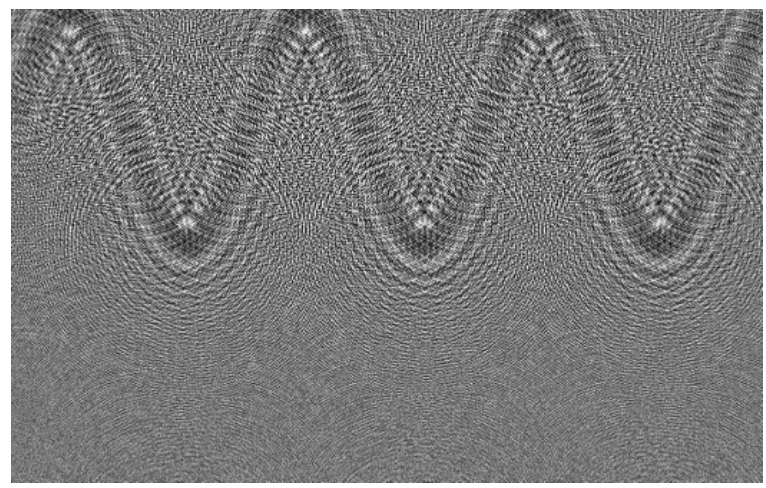

Fig. 15.21. Computed hologram of the object shown in Fig. 15.20 created by (15.7) for a LCoS SLM

It is observed that the reconstruction quality is satisfactory even if another SLM, than the one intended during the computation, is used during the reconstruction.

Up to now, the presented experiments are based on monochromatic wave propagation. As the next step, we evaluate the performance of the Fresnel hologram computation algorithm for colour holograms. Images of off-axis colour Fresnel hologram (Fig. 15.26) of the 3DTV Project Logo (Fig. 15.25)

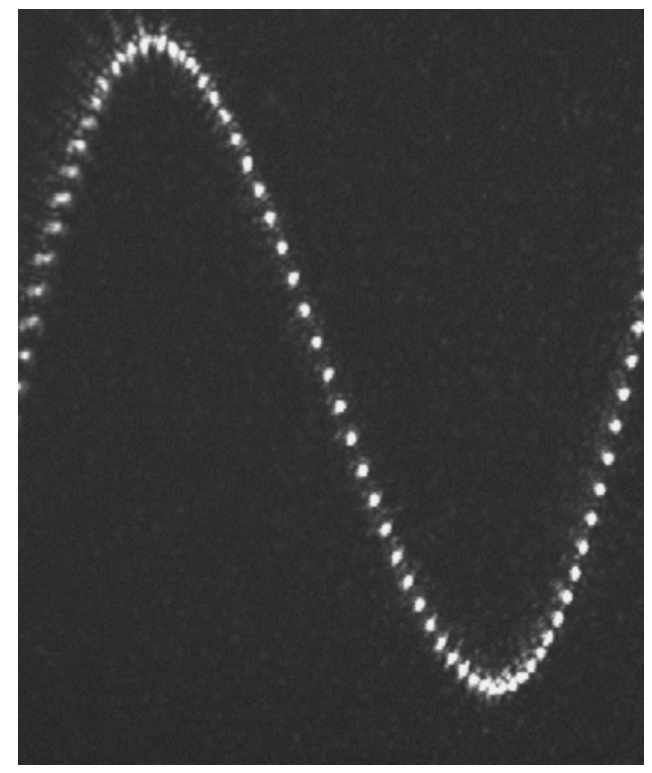

Fig. 15.22. A portion of the optically reconstructed image by the LCoS SLM of the "sine-wave" object shown in Fig. 15.20 from the hologram shown in Fig. 15.21 


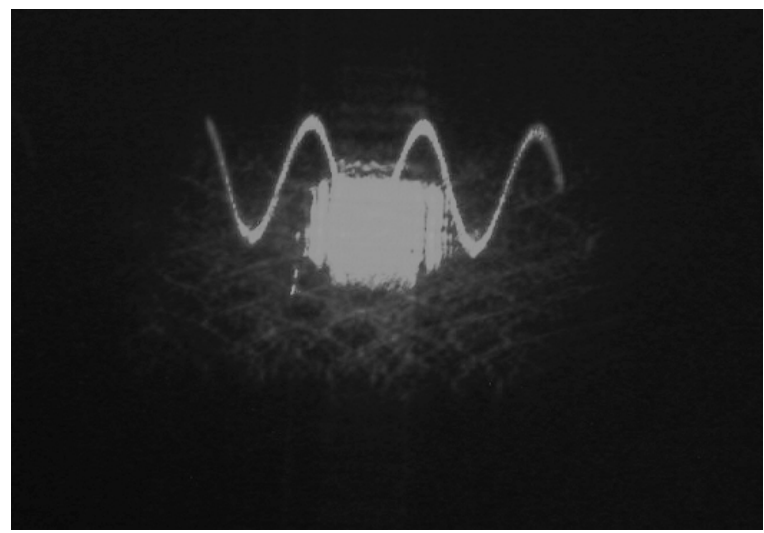

Fig. 15.23. Optically reconstructed image by the LC SLM of the "sine-wave" object shown in Fig. 15.20 from the hologram shown in Fig. 15.21. The bright rectangle obstructing the image is the zeroth order (First published by Springer [12])

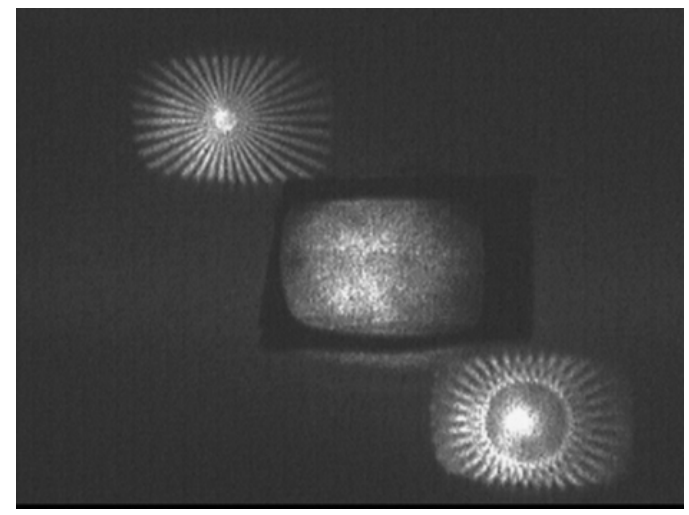

Fig. 15.24. Optically reconstructed image from the off-axis hologram shown in Fig. 15.9 by the LCoS SLM (First published by Springer [12])

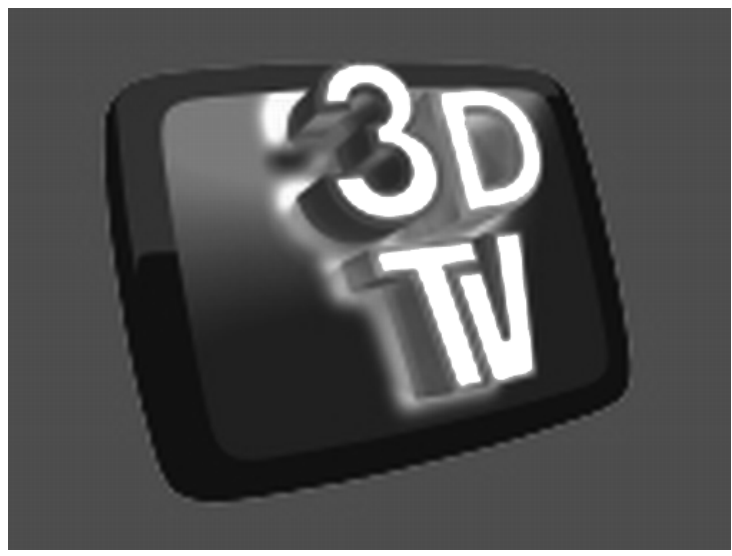

Fig. 15.25. 3DTV Project Logo as a colour object 


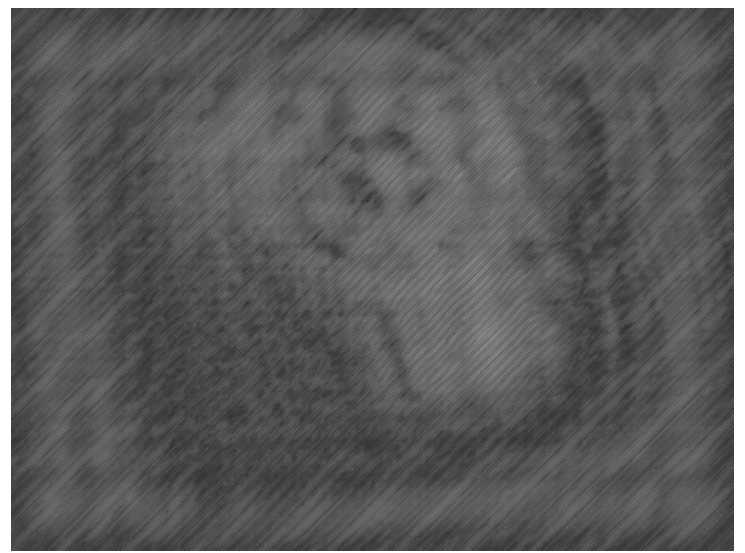

Fig. 15.26. Colour hologram of the object in Fig. 15.25 (First published by Springer $[12])$

are reconstructed using the LC SLM whose resolution is $1280 \times 720$ pixels. Optical and computer simulation based reconstructions are illustrated in Figs. 15.27 and 15.28, respectively. The colour images cannot be presented here because of black-and-white printing process. The object (Fig. 15.25) is split into its R, G, B colour components. The R-component is shown in Fig. 15.29 in gray scale. Then separate holograms are computed for each component using (15.6). The hologram for the R-component is shown in Fig. 15.30.

The colour hologram in Fig. 15.26 is generated by numerically superposing the calculated holograms for the R, G and B components, using their respective colours during the superposition. The reconstruction from the hologram corresponding to the R component by the LC SLM using a red laser is shown

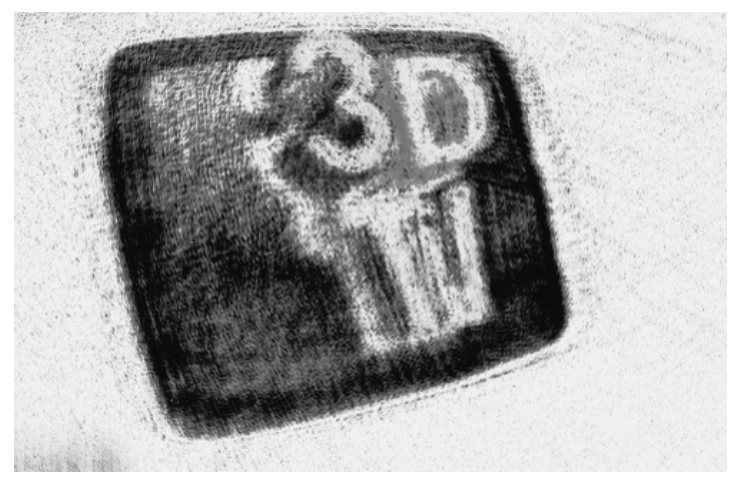

Fig. 15.27. Reconstructed 3DTV Project Logo by superposition of optically reconstructed from RGB components of Fig. 15.26 (First published by Springer [12]) 


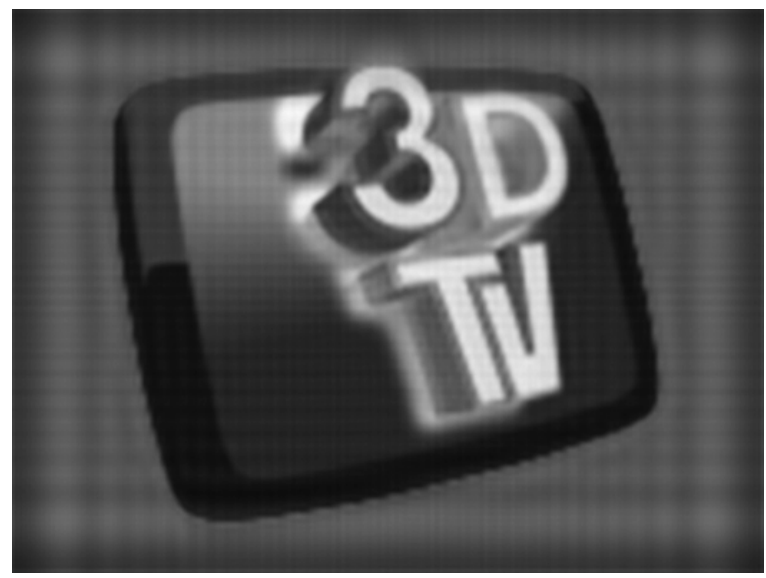

Fig. 15.28. Numerically reconstructed 3DTV Logo from Fig. 15.26 (First published by Springer [12])

in Fig. 15.31. The image is captured by a digital camera. Similar SLM reconstructions are carried out also for the $\mathrm{G}$ and $\mathrm{B}$ holograms and each reconstruction is captured by the digital camera. The captured images for each colour component are then combined, numerically, to yield the colour image in Fig. 15.27. Numerical reconstructions, instead of optical SLM reconstructions, are also carried out for comparison, and the result is shown in Fig. 15.28. Various papers on colour CGH can be found in the literature [35, 36, 37, 36, 39]. Consequently, we can say that LC SLMs can be used to recontruct the colour holograms.

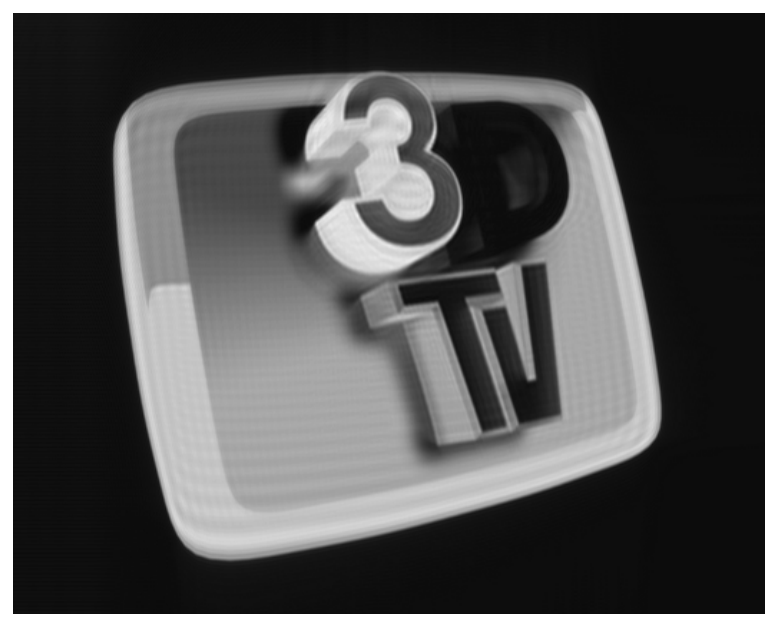

Fig. 15.29. Red-component of the colour object shown in Fig. 15.25 


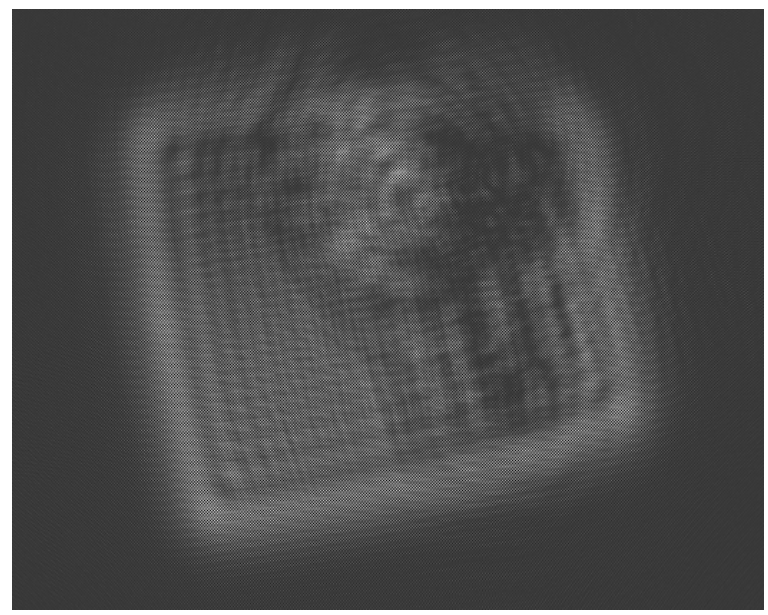

Fig. 15.30. Computed hologram of the Red component

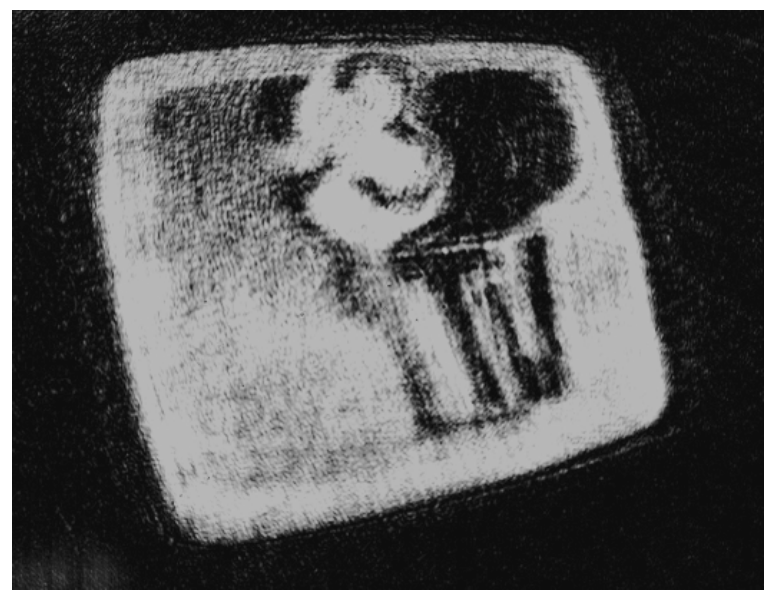

Fig. 15.31. Optically reconstructed Red-component of the hologram given in Fig. 15.30

\subsection{Conclusion}

SLMs are promising devices for dynamic holographic displays. The quality of reconstructed images using the currently available SLMs is promising, but not satisfactory. SLM pixel size of about $0.4-0.6 \mu \mathrm{m}$ is needed to write good quality holograms. Nowadays commercially available SLMs, which could be used as a media for holograms, have a pixel size of $7-8 \mu \mathrm{m}$ and the number of pixels can go up to $3840 \times 2048$. Pixel size depends on photolithographic and microelectronic technologies. So, about 10 times real or virtual improvement of pixel size is needed. 
Algorithms used for CGH are also important. They must be efficient and fast enough to be able to work in real time. Moreover, the resolution of the reconstructed 3D scene has to be equivalent or better than that of the commonly used 2D displays. At the moment algorithms used to compute holograms are efficient for real time processing only of quite low resolution objects $[13,21,40]$.

The algorithms based on the Fresnel-Kirchhoff diffraction formula and Rayleigh-Sommerfeld diffraction integral provide similar reconstructed patterns when the distance along the optical axis is around $0.8 \mathrm{~m}$. The holograms are calculated with a resolution of $1280 \times 720$ pixels for an object with the same resolution using a $3.6 \mathrm{GHz}$ personal computer. The achieved computing speed of $3.25 \times 10^{-5} \mathrm{~s} /$ point is better than the published results (about $10^{-4} \mathrm{~s} /$ point) for several algorithms $[21,31,40]$. Naturally, the computational complexity is three times higher, than the monochrome holographic display, for colour holography.

A match between the parameters of the SLM used during the reconstruction, and the computed hologram is desirable for better quality. However, conducted experiments show that reconstructions using different SLMs could be satisfactory, too.

SLMs has the potential to be used for color holographic displays, as well.

\section{Acknowledgements}

The authors thank Stefan Osten, Holoeye Photonics AG, Berlin, Germany for providing the amplitude and phase modulation characteristics of Holoeye SLMs.

This work is supported by EC within FP6 under Grant 511568 with acronym 3DTV.

\section{References}

1. Onural L., Bozdagi G., Atalar A., New high-resolution display device for holographic three-dimensional video: principles and simulations, Optical Engineering, Vol. 33, No. 3, 835-844, 1994.

2. Fukaya N., Maeno K., Sato K., Honda T., Improved electroholographic display using liquid crystal devices to shorten the viewing distance with both-eye observation, Optical Engineering, Vol. 35, No. 6, 1545-1549, 1996.

3. Huignard J. P., Spatial light modulators and their applications, J. Optics, Vol. 18, No. 4, 181-186, 1987.

4. Bleha W. P., Sterling R. D., D-ILA ${ }^{\text {TM }}$ Technology for high resolution projection displays, JVC ILA Technology Group, 20984 Bake Parkway, Suite 102, Lake Forest CA 92630 USA.

5. Lucente M., Computational holographic bandwidth compression, IBM Systems Journal, Vol. 35, No. 3/4, 349-365, 1996. 
6. Yang H. and Lu M., Nematic LC modes and LC phase gratings for reflective spatial light modulators, IBM Journal of Research and Development, Vol. 42, No. 3/4, 401-410, 1998.

7. Mishina T., Okui M., Okano F., Viewing-zone enlargement method for sampled hologram that uses high-order diffraction, Applied Optics, Vol. 41, No. 8, 1489-1499, 2002.

8. Mishina T., Okano F., Yuyama I., Time-alternating method based on singlesideband holography with half-zone-plate processing for the enlargement of viewing zones, Applied Optics, Vol. 38, No. 17, 3703-3713, 1999.

9. Born M., Wolf E., "Principles of Optics", Pergamon Press, New York, 4th ed., 1970.

10. Cameron C. D., Pain D. A., Stanley M., Slinger C. W., Computational challenges of emerging novel true 3D holographic displays, in Critical Technologies for the Future of Computing, S. Bains, L. J. Irakliotis, eds., Proceedings of SPIE, Vol. 4109, 129-140, 2000.

11. Abookasis D., Rosen J., Three types of computer-generated holograms synthesized from multiple angular viewpoints of a three-dimensional scene, Applied Optics, Vol. 45, No. 25, 6533-6538, 2006.

12. Kovachev M., Ilieva R., Onural L., Esmer G. B., Reyhan T., Benzie P., Watson J., Mitev E., "Reconstruction of Computer Generated Holograms by Spatial Light Modulators", Proceedings IW MRCS 2006, Istanbul, Turkey, LNCS 4105, 706-713, 2006.

13. Fukushima S., Kurokawa T., Ohno M., Real-time hologram construction and reconstruction using a high-resolution spatial light modulators, Applied Physics Letters, Vol. 58, 787-789, 1991.

14. Lucke R. L., Rayleigh-Sommerfeld diffraction and Poisson's spot, European Journal of Physics, Vol. 27, 193-204, 2006.

15. Amuasi H., The Mathematics of Holography, Essays Towards the AIMS Postgradute Diploma (2003/2004), African Institude for Mathematical Sciences, 2004. http://www.aims.ac.za/resources/archive/2003/henryessay2.0.pdf

16. Goodman J. W. "Introduction to Fourier Optics", Roberts \& Company Publisher, U.S., 3rd edition, 2004.

17. Asundi A., Singh V. R., Sectioning of amplitude images in digital holography, Measurement Science and Technology, Vol. 17, 75-78, 2006.

18. Janda M., Hanak I., Skala V., Digital HPO Hologram Rendering Pipeline, EUROGRAPHIC short papers conference, Proceedings, 81-84, 2006.

19. Ito T., Okano K., Color electroholography by three colored reference lights simultaneously incident upon one hologram panel, Optics Express, Vol. 12, No. 18, 4320-4325, 2004.

20. Ito T., Holographic reconstruction with a $10-\mu \mathrm{m}$ pixel-pitch reflective liquidcrystal display by use of a light-emitting diode reference light, Optics Letters, Vol. 27, No. 16, 1406-1408, 2002.

21. Lucente M., Optimization of hologram computation for real-time display, SPIE Proceeding, "Practical Holography VI", Vol. 1667, 32-43, 1992.

22. Leibling M., Unser M., Autofocus for digital Fresnel holograms by use of a Fresnelet-sparsity criterion, Journal Optical Society of America A, Vol. 21, No. 12, 2424-2430, 2004.

23. Choi K., Kim H., Lee B., Synthetic phase holograms for auto-stereoscopic image displays using a modified IFTA, Optics Express, Vol. 12, No. 11, 2454-2461, 2004 . 
24. Leibling M., Thierry Blu, Unser M., Complex-wave retrieval from a single offaxis hologram, Journal Optical Society of America A, Vol. 21, No. 3, 367-377, 2004.

25. Plesniak W., Incremental update of computer-generated holograms, Optical Engineering, Vol. 42, No. 6, 1560-1571, 2003.

26. Mezouari S., Harvey A. R., Validity of Fresnel and Fraunhofer approximations in scalar diffraction, Journal of Optics A: Pure Applied Optics, Vol. 5, S86-S91, 2003.

27. Grilli S., Ferraro P., De Nicola S., Finizio A., Pierattini G., Meucci R., Whole optical wavefield reconstruction by digital holography, Optics Express, Vol. 9, No. 6, 294-302, 2001.

28. Kajiki Y., Ueda H., Tanaka K., Okamoto H., Shimizu E., Cylindrical large computer-generated holograms and hidden-point removal process, Proceedings of SPIE, Vol. 2652, 29-35, 1996.

29. Sherman G. C., Application of the convolution theorem to Rayleigh's integral formulas, Journal Optical Society of America, Vol. 57, 546-547, 1967.

30. Lalor E., Conditions for the validity of the angular spectrum of plane waves, Journal Optical Society of America, Vol. 58, 1235-1237, 1968.

31. Ahrenberg L., Benzie P., Magnor M., Watson J., Computer generated holography using parallel commodity graphics hardware, Optics Express, Vol. 14, 7636-7641, 2006.

32. Kries T., Hologram reconstruction using a digital micromirror device, Optical Engineering, Vol. 40, No. 6, 926-933, 2001.

33. Schnars U., Juptner W., Direct recording of holograms by a CCD target and numerical reconstructions, Applied Optics, Vol. 33, No. 2, 179-181, 1994.

34. Vdovin G., LightPipes: beam propagation toolbox, OKO Technologies, The Netherlands, 1999.

35. Shimobaba T., Ito T., A color holographic reconstruction system by time division multiplexing with reference lights of laser, Optical Review, Vol. 10, No. 5, 339-341, 2003.

36. Choi K., Kim H., Lee B., Full-color autostereoscopic 3D display system using color-dispersion-compensated synthetic phase holograms, Optics Express, Vol. 12, No. 21, 5229-5236, 2004.

37. Suh H. H., Color-image generation by use of binary-phase holograms, Optics Letters, Vol. 24, No. 10, 661-663, 1999.

38. Sando Y., Itoh M., Yatagai T., Full-color computer-generated holograms using 3-D Fourier spectra, Optics Express, Vol. 12, No. 25, 6246-6251, 2004.

39. Sando Y., Itoh M., Yatagai T., Color computer-generated holograms from projection images, OSA, Optics Express, Vol. 12, No. 11, 2487-2493, 2004.

40. Munjuluri B., Huebschman M., Garner H., Rapid hologram updates for realtime volumetric information display, Applied Optics, Vol. 44, No. 24, 5076-5085, 2005.

41. Wernicke G., Krueger S., Kamps J., Gruber H., Demoli N., Duer M., Teiwes S., Application of a liquid crystal display spatial light modulator system as dynamic diffractive element and in optical image processing, Journal of Optical Communications, Vol. 25, No. 4, 141-148, 2004.

42. HoloEye Spatial Light Modulators. http://www.holoeye.com/spatial_light_ modulators-technology.htm

43. Onural L., Exact analysis of the effects of sampling of the scalar diffraction field, Journal Optical Society of America A, Vol. 24, No. 2, 359-367, 2007. 